Research Paper

\title{
The impact of SPARC expression on the survival of pancreatic ductal adenocarcinoma patients after curative resection
}

\author{
Masaaki Murakawa ${ }^{1}$, Toru Aoyama 4, Yohei Miyagi², Satoshi Kobayashi³, Makoto Ueno ${ }^{3}$, Manabu \\ Morimoto ${ }^{3}$, Masakatsu Numata ${ }^{4}$, Naoto Yamamoto ${ }^{1}$, Hiroshi Tamagawa ${ }^{4}$, Norio Yukawa ${ }^{4}$,Yasushi Rino ${ }^{4}$, \\ Munetaka Masuda ${ }^{4}$, Soichiro Morinaga ${ }^{1}$ \\ 1. Department of Gastrointestinal Surgery, Kanagawa Cancer Center, Yokohama, Japan \\ 2. Molecular Pathology and Genetics Division, Kanagawa Cancer Center Research Institute \\ 3. Department of Hepatobiliary Pancreatic Oncology, Kanagawa Cancer Center \\ 4. Department of Surgery, Yokohama City University \\ Masaaki Murakawa and Toru Aoyama equally contributed this article. \\ $\triangle$ Corresponding author: Masaaki Murakawa, Department of Gastrointestinal Surgery, Kanagawa Cancer Center, 2-3-2 Nakao, Asahi-ward, Yokohama city, \\ 241-8515, Japan TEL: 81-45-520-2222, E-mail: t176063g@yokohama-cu.ac.jp \\ (C) Ivyspring International Publisher. This is an open access article distributed under the terms of the Creative Commons Attribution (CC BY-NC) license \\ (https://creativecommons.org/licenses/by-nc/4.0/). See http://ivyspring.com/terms for full terms and conditions.
}

Received: 2018.07.20; Accepted: 2018.11.07; Published: 2019.01.01

\begin{abstract}
Background: The predictive roles of secreted protein acidic and rich in cysteine (SPARC) in pancreatic ductal adenocarcinoma (PDAC) patients after curative resection have not been clarified. We investigated the correlations between the SPARC expression and the postoperative prognosis.

Methods: We retrospectively analyzed the clinical data from consecutive patients who underwent curative resection for pancreatic cancer in our institution from 2005 to 2014. Stromal SPARC expression was analyzed by immunohistochemistry on tumor tissue microarrays (TMAs) from the patients.

Results: A total of 179 patients were enrolled to this study. The median follow-up period of the present study was 62.1 months. Seventy patients had positive SPARC expression (39.1\%). There were no significant differences between the positive SPARC-positive group and the SPARC-negative group. In the survival analysis, there was a significant difference between the SPARC-positive and SPARC-negative groups in the 5 -year overall survival (OS) rates after surgery, which were $8.1 \%$ and $19.8 \%$, respectively $(P=0.0316)$. A univariate analysis showed that the SPARC expression, size of tumor, lymph node metastasis, and residual tumor were possible prognostic factors. A multivariate analysis showed that the SPARC expression (hazard ratio [HR]: 1.44, 95\% confidence interval [Cl]: 1.017-2.051), lymph node metastasis (HR: $2.019,95 \% \mathrm{Cl}$ : 1.318-3.091), and residual tumor (HR: $1.648,95 \% \mathrm{Cl}: 1.132-2.401$ ) were independent prognostic factors.
\end{abstract}

Conclusions: The stromal SPARC expression in resectable pancreatic cancer patients might be useful as a prognostic marker.

Key words: pancreatic cancer; secreted protein acidic and rich in cysteine; prognostic factor

\section{Introduction}

Pancreatic ductal adenocarcinoma (PDAC) is a lethal disease found worldwide. The GLOBOCAN 2012 estimated that pancreatic cancer is the seventh leading cause of cancer death ${ }^{1}$. Other reports have also mentioned that pancreatic cancer will become the second leading cause of cancer-related death in the United States by $2030^{2}$. The only chance for a cure is to receive a diagnosis at the resectable stage and to 
undergo multidisciplinary therapy: complete surgical resection and perioperative adjuvant treatment ${ }^{3-5}$.

However, despite technical advances in surgical resection, perioperative management, and multi-drug combination chemotherapy, the survival improvement is insufficient compared with most other cancers. For example, the Japan Adjuvant Study Group of Pancreatic Cancer showed that S-1 (oral fluorouracil) adjuvant chemotherapy improved the disease-free survival (DFS) and overall survival (OS) for completely resected pancreatic cancer patients with a 5-year survival rate of $44.1 \% 5$. However, recurrence was observed in $72 \%$ of patients with curatively resected pancreatic cancer. A more effective therapy is therefore urgently needed.

Secreted protein acidic and rich in cysteine (SPARC) is an extracellular protein that plays roles in cancer cell migration, proliferation, angiogenesis, matrix cell adhesion, and tissue remodeling 6 . Pancreatic cancer is a tumor consisting of abundant stroma and an extracellular matrix and tumor-stroma cells are related to tumor progression, promoting carcinogenesis and treatment resistance ${ }^{7-9}$. In recent studies, SPARC has been found to localize to the tumor stroma and to be overexpressed in various cancers, such as breast, lung, and melanoma. Although many studies have evaluated the significance of SPARC expression, its role in carcinogenesis remains unclear ${ }^{10-14}$. Stromal SPARC expression is reported to be associated with a poor prognosis. However, the most important issue at present is that a clinical standard of SPARC expression has not been established.

In this study, we investigated the SPARC expression of consecutive patients who underwent curative resection and evaluated the relationship among the SPARC expression, clinicopathological parameters, and survival.

\section{Patients and Methods}

\section{Patients}

The patients were selected from the medical records of consecutive patients who underwent pancreatic surgery at Kanagawa Cancer Center from 2005 to 2014. Eligible patients were (1) pathologically diagnosed with PDAC based on the definitions of the UICC TNM 7th edition ${ }^{15}$ and (2) patients who underwent $\mathrm{R} 0$ or $\mathrm{R} 1$ resection. The resected specimens were examined histopathologically and staged according to the UICC TNM 7th edition. Patients with pathologically proven Stage IV disease, such as lymph node metastasis to para-aortic lymph nodes, and those who underwent $\mathrm{R} 2$ resection were excluded from this study.

\section{Surgical procedure}

All of the operations were performed by four surgeons from the pancreatic unit. All pancreatic surgeries were performed in accordance with standardized procedures that have been described elsewhere. In brief, in cases of pancreaticoduodenectomy (PD), we performed subtotal stomach-preserving pancreaticoduodenectomy as the standard procedure. The lymph node groups that were resected en bloc included the anterior pancreatic duodenal lymph nodes, the posterior pancreatic duodenal lymph nodes, nodes in the lower hepatoduodenal ligament, and nodes along the right lateral aspect of the superior mesenteric artery and vein. In cases of distal pancreatectomy (DP), lymph node dissection was performed in the region of the celiac trunk and the superior mesenteric artery and vein, as well as behind the pancreas along the left side of the renal vein and the left adrenal gland.

\section{Adjuvant chemotherapy}

S-1 treatment was started within 10 weeks after surgery. The patients received 80 to $120 \mathrm{mg} /$ day for 4 weeks followed by 2 weeks of rest, and treatment was continued for 6 months. The doses were modified in accordance with the JASPAC-01 trial5; when adverse reactions appeared, the dose was reduced from 120 by $20 \mathrm{mg} /$ day. Gemcitabine treatment was started within eight weeks of surgery and continued for six months. The patients received a weekly dose of 1,000 $\mathrm{mg} / \mathrm{m} 2$ for 3 weeks, followed by 1 week of rest. Adjuvant chemotherapy was discontinued when the patient showed disease recurrence or adverse events that were uncontrollable even by dose modification or the temporary withdrawal.

\section{Follow-up}

Patients were followed up at outpatient clinics for five years after finishing surgical resection and adjuvant chemotherapy. The CEA and CA19-9 levels were evaluated at least every three months for five years. Patients underwent computed tomography every three months during the first three years after surgery and then every six months until five years after surgery.

\section{The immunohistochemical analysis of the SPARC expression}

Slides from all resection specimens were retrieved from the archive and reviewed by expert gastrointestinal pathologists. The H\&E slide with the highest tumor cell density and the deepest tumor infiltration was selected. Hematoxylin and eosin-stained slides of the representative PDAC 
region were reviewed, and the corresponding formalin-fixed, paraffin-embedded tissue blocks were selected for use in a tissue microarray. The SPARC expression was evaluated using purified rabbit polyclonal antibody, which was purchased from EMD Millipore (Temecula, CA, USA). The intensity of stromal SPARC staining was scored as follows: grade 0 , not stained; grade 1 , faintly stained; grade 2 , weakly stained; and grade 3, stained as strongly in stroma. (Figure 1). The immunohistochemical evaluation of the SPARC expression was independently confirmed by two observers (M.M. and Y.M.), and a consensus was reached by joint review.

\section{Evaluations and statistical analyses}

The significance of the correlations between the SPARC expression and clinicopathological parameters was determined using Fisher's exact test or a $\chi^{2}$ test. The OS was defined as the period between surgery and death. The data of the patients who had experienced no events were censored at the date of the final observation. The OS curves were calculated using the Kaplan-Meier method and compared using the log-rank test. The univariate and multivariate survival analyses were performed using Cox's proportional hazards model. P values of $<0.05$ were considered statistically significant. The survival data were obtained from hospital records or from the city registry system. The SPSS software program (v11.0 J Win, SPSS, Chicago, IL, USA) was used for all of the statistical analyses. This study was approved by the IRB Committee of the Kanagawa Cancer Center.

\section{Results}

\section{Patients}

A total of 201 patients underwent surgical resection between 2005 and 2014, and 179 patients were eligible for this study. The median age was 68 years (range, $40-86$ years); 97 patients were male, and 82 were female. One hundred and thirteen patients underwent pancreaticoduodenectomy, 54 distal pancreatectomy, and 12 total pancreatectomy. The median follow-up period was 62.1 months (range, 3.4-122.4 months). As adjuvant chemotherapy after resection, 79 patients received gemcitabine therapy, 68 patients S- 1 therapy, 4 patients gemcitabine and S- 1 combination therapy, and 28 patients no adjuvant chemotherapy.

\section{Immunohistochemical analyses and patients' background characteristics}

The representative results of immunohistochemical staining for SPARC in PDAC tissue stroma are shown in Figure 1. Immunoreactivity was mostly observed in the stroma of pancreatic cancer tissue. Of the 179 tumor samples, $70(39.1 \%)$ showed positive staining. Seventy patients were thus classified to the SPARC-positive group and 109 to the SPARC-negative group. Table 1 compares the patient background characteristics between these two groups. There were no significant differences between the two groups in gender, age, tumor location, size of tumor, implemented adjuvant chemotherapy, and pathological status.

\section{Recurrence-free survival (RFS) and OS analyses}

The 5-year OS rates in the SPARC-positive and SPARC negative patients were $29.7 \%$ and $8.1 \%$, respectively ( $p=0.032$; Figure 2$)$. The 5 -year RFS rates in the SPARC-positive and SPARC negative patients were $11.8 \%$ and $14.1 \%$, respectively $(\mathrm{p}=0.139$; Figure $3)$.

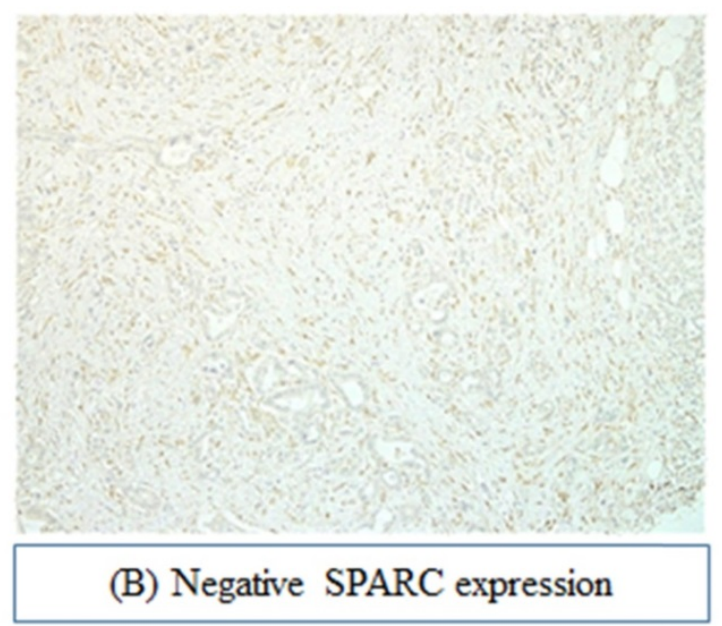

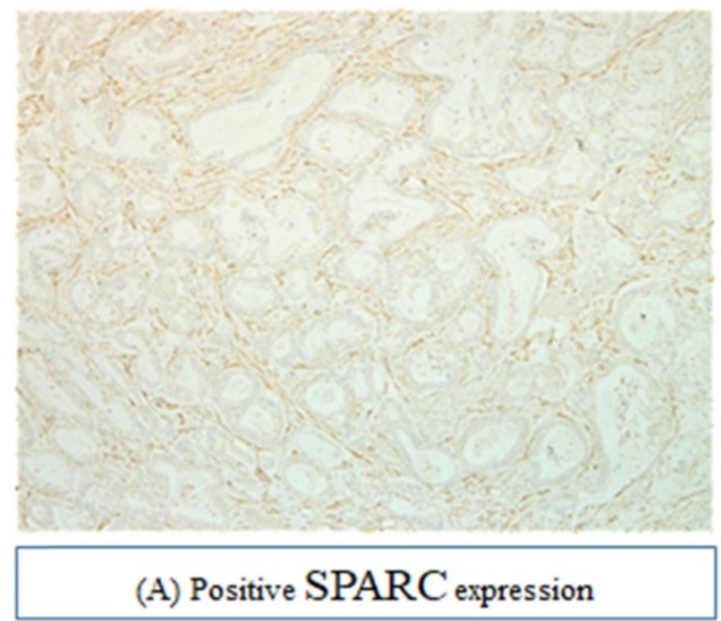

SPARC antibody (Santa Cruz Biotechnology, Santa Cruz, CA)

Figure 1: Immunohistochemical analysis of pancreatic ductal carcinoma specimens for SPARC expression. 
Table 1. A comparison of the patient background between the SPARC-positive and SPARC-negative groups.

\begin{tabular}{|c|c|c|c|c|c|c|}
\hline & & $\begin{array}{l}\text { Patients p } \\
\text { SPARC (n }\end{array}$ & $\begin{array}{l}\text { itive for } \\
\text { 70) }\end{array}$ & $\begin{array}{l}\text { Patients ne } \\
\text { SPARC }(n=\end{array}$ & $\begin{array}{l}\text { ative for } \\
\text { 09) }\end{array}$ & $\begin{array}{l}P \\
\text { value }\end{array}$ \\
\hline $\begin{array}{l}\text { Age (median, } \\
\text { years) }\end{array}$ & & $67(44-81)$ & & $66(51-81)$ & & 0.266 \\
\hline \multirow[t]{2}{*}{ Gender } & Male & 37 & $52.9(\%)$ & 60 & $55(\%)$ & 0.89 \\
\hline & Female & 33 & $47.1(\%)$ & 49 & $45(\%)$ & \\
\hline \multirow[t]{3}{*}{ Surgical procedure } & $\mathrm{PD}$ & 40 & $57.1(\%)$ & 73 & $67(\%)$ & 0.14 \\
\hline & $\mathrm{DP}$ & 26 & $37.1(\%)$ & 28 & $25.7(\%)$ & \\
\hline & $\mathrm{TP}$ & 4 & $5.7(\%)$ & 8 & $7.3(\%)$ & \\
\hline $\begin{array}{l}\text { Size of tumor } \\
\text { (median, mm) }\end{array}$ & & $35(12-90)$ & & $35(5-105)$ & & 0.718 \\
\hline \multirow[t]{4}{*}{ Pathological type } & tub1 & 22 & $31.4(\%)$ & 49 & $45(\%)$ & 0.708 \\
\hline & tub2 & 35 & $50(\%)$ & 39 & $35.8(\%)$ & \\
\hline & por & 9 & $12.9(\%)$ & 15 & $13.8(\%)$ & \\
\hline & others & 4 & $5.7(\%)$ & 6 & $5.5(\%)$ & \\
\hline \multirow{4}{*}{$\begin{array}{l}\text { Pathological } \\
\text { T factor }\end{array}$} & $\mathrm{T} 1$ & 2 & $2.9(\%)$ & 6 & $5.5(\%)$ & 0.163 \\
\hline & $\mathrm{T} 2$ & 1 & $1.4(\%)$ & 4 & $3.7(\%)$ & \\
\hline & T3 & 62 & $88.6(\%)$ & 97 & $89(\%)$ & \\
\hline & $\mathrm{T} 4$ & 5 & $7.1(\%)$ & 2 & $1.8(\%)$ & \\
\hline \multirow{2}{*}{$\begin{array}{l}\text { Pathological } \\
\mathrm{N} \text { factor }\end{array}$} & No & 22 & $31.4(\%)$ & 30 & $27.5(\%)$ & 0.694 \\
\hline & N1 & 48 & $68.6(\%)$ & 79 & $72.5(\%)$ & \\
\hline \multirow[t]{2}{*}{ Residual tumor } & R0 & 52 & $74.3(\%)$ & 82 & $75.2(\%)$ & 0.972 \\
\hline & $\mathrm{R} 1$ & 18 & $25.7(\%)$ & 27 & $24.8(\%)$ & \\
\hline \multirow{2}{*}{$\begin{array}{l}\text { Pathological } \\
\text { Stage }\end{array}$} & IA & 2 & $2.9(\%)$ & 6 & $5.5(\%)$ & 0.85 \\
\hline & IB & 0 & $0(\%)$ & 1 & $0.9(\%)$ & \\
\hline
\end{tabular}

\begin{tabular}{|c|c|c|c|c|c|c|}
\hline & IIA & 19 & $27.1(\%)$ & 23 & $21.1(\%)$ & \\
\hline & IIB & 45 & $64.3(\%)$ & 77 & $70.6(\%)$ & \\
\hline & III & 4 & $5.7(\%)$ & 2 & $1.8(\%)$ & \\
\hline \multirow{2}{*}{$\begin{array}{l}\text { Adjuvant } \\
\text { chemotherapy }\end{array}$} & yes & 60 & $85.7(\%)$ & 91 & $83.5(\%)$ & 0.681 \\
\hline & no & 10 & $14.3(\%)$ & 18 & $16.5(\%)$ & \\
\hline
\end{tabular}

A univariate analysis of the OS showed that the tumor size, pathological $\mathrm{N}$ factor, resection margin, and SPARC expression were independent factors affecting the survival. Multivariate analysis showed that the pathological $\mathrm{N}$ factor, resection margin, and SPARC expression were independent factors affecting the survival (Table 2). In addition, the univariate analysis for recurrence showed that the tumor size, pathological $\mathrm{N}$ factor, and resection margin were independent factors affecting the recurrence. A multivariate analysis showed that the pathological $\mathrm{N}$ factor and resection margin were independent factors affecting the recurrence (Table 3).

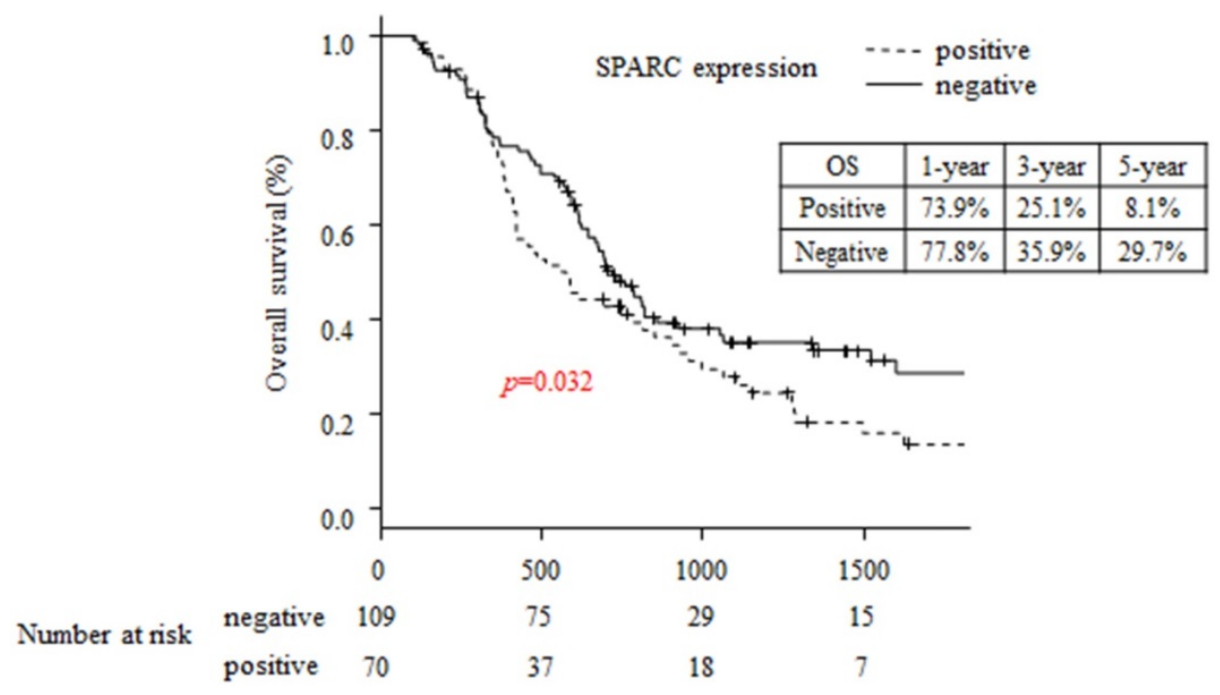

Figure 2: Comparison of overall survival between the SPARC-positive and SPARC-negative groups.

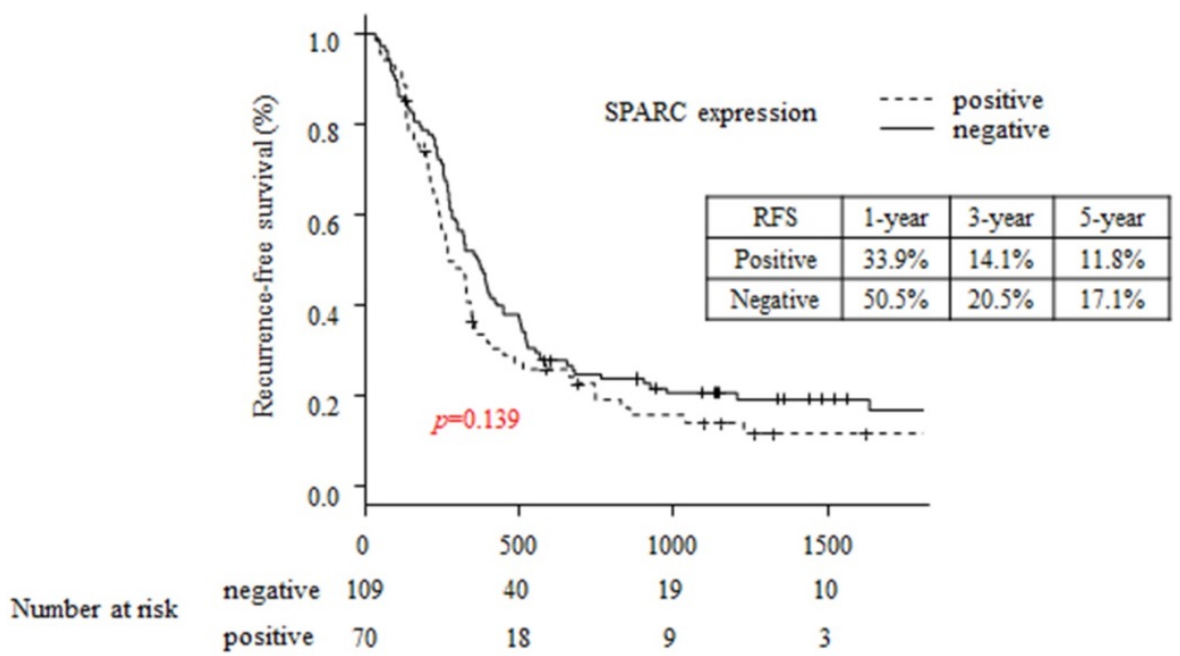

Figure 3: Comparison of recurrence-free survival between the SPARC-positive and SPARC-negative groups. 
Table 2. Univariate and multivariate analyses of risk factors for the recurrence-free survival

\begin{tabular}{|c|c|c|c|c|c|c|c|}
\hline & & \multicolumn{3}{|c|}{ Univariate analysis } & \multicolumn{3}{|c|}{ Multivariate analysis } \\
\hline & & HR & $95 \% \mathrm{CI}$ & P value & $H R$ & $95 \% \mathrm{CI}$ & P value \\
\hline \multirow[t]{2}{*}{ Age (years) } & $<68$ & 1.000 & $0.7472-1.487$ & 0.764 & & & \\
\hline & $\geq 68$ & 1.054 & & & & & \\
\hline \multirow[t]{2}{*}{ Gender } & Male & 1.000 & $0.9304-1.837$ & 0.1225 & & & \\
\hline & Female & 1.307 & & & & & \\
\hline \multirow{2}{*}{$\begin{array}{l}\text { Tumor } \\
\text { location }\end{array}$} & Head & 1.000 & $0.8459-1.787$ & 0.2788 & & & \\
\hline & $\begin{array}{l}\text { Body and } \\
\text { tail }\end{array}$ & 1.230 & & & & & \\
\hline \multirow[t]{2}{*}{ Size of tumor } & $<35 \mathrm{~mm}$ & 1.000 & $1.001-1.954$ & 0.04959 & 1.000 & $0.959-1.877$ & 0.086 \\
\hline & $\geq 35 \mathrm{~mm}$ & 1.398 & & & 1.342 & & \\
\hline \multirow{2}{*}{$\begin{array}{l}\text { Pathological } \\
\mathrm{N} \text { factor }\end{array}$} & N0 & 1.000 & $1.212-2.649$ & 0.00343 & 1.000 & $1.343-2.868$ & 0.0005 \\
\hline & N1 & 1.792 & & & 1.963 & & \\
\hline \multirow{2}{*}{$\begin{array}{l}\text { Resection } \\
\text { margin }\end{array}$} & R0 & 1.000 & $1.018-2.197$ & 0.04049 & 1.000 & $1.113-2.305$ & 0.0111 \\
\hline & R1 & 1.495 & & & 1.602 & & \\
\hline \multirow{2}{*}{$\begin{array}{l}\text { SPARC } \\
\text { expression }\end{array}$} & Positive & 1.000 & $0.866-1.707$ & 0.257 & & & \\
\hline & Negative & 1.216 & & & & & \\
\hline
\end{tabular}

Table 3. Univariate and multivariate analyses of risk factors for the overall survival

\begin{tabular}{llllllll}
\hline & \multicolumn{5}{c}{ Univariate analysis } & \multicolumn{3}{c}{ Multivariate analysis } \\
\cline { 3 - 8 } & & $H R$ & $95 \%$ CI & P value & HR & $95 \%$ CI & P value \\
\hline Age (years) & $<68$ & 1.000 & $0.762-1.523$ & 0.574 & & & \\
& $\geq 68$ & 1.077 & & & & & \\
Gender & Male & 1.000 & $0.894-1.792$ & 0.138 & & & \\
& Female & 1.266 & & & & & \\
Tumor & Head & 1.000 & $0.901-1.959$ & 0.155 & & & \\
location & Body and & 1.328 & & & & & \\
& tail & & & & & & \\
Size of tumor & $<35 \mathrm{~mm}$ & 1.000 & $0.484-8.107$ & 0.0235 & 1.000 & $0.989-2.077$ & 0.058 \\
& $\geq 35 \mathrm{~mm}$ & 1.454 & & & 1.433 & & \\
Pathological & N0 & 1.000 & $1.328-3.073$ & 0.00078 & 1.000 & $1.232-2.993$ & 0.004 \\
N factor & N1 & 2.020 & & & 1.920 & & \\
Resection & R0 & 1.000 & & 0.00241 & 1.000 & $1.158-2.514$ & 0.007 \\
margin & R1 & 1.741 & $1.197-2.532$ & & 1.707 & & \\
SPARC & Positive & 1.000 & & 0.0432 & 1.000 & $1.052-2.164$ & 0.025 \\
expression & Negative & 1.461 & $1.03-2.072$ & & 1.509 & & \\
\hline [HR: Hazard ratio CI: confidential interval] & & &
\end{tabular}

\section{Discussion}

The aim of the present study was to evaluate the clinical impact of the stromal SPARC expression as a prognostic factor in pancreatic adenocarcinoma patients who underwent curative resection. The major findings were that stromal SPARC expression was observed in almost $40 \%$ of pancreatic adenocarcinoma patients who underwent curative resection, and this expression was an independent prognostic factor. Our results suggest that current adjuvant chemotherapy is insufficient, especially in patients with high SPARC expression. These patients should be a target group for clinical trials of novel treatments.

Many investigators have examined the impact of SPARC overexpression in patients with PDAC11-13,16,18-24. These studies have reported that SPARC is highly expressed in $10 \%-60 \%$ of PDAC patients. However, the measurement of SPARC expression was not standardized in those previous studies, and the background of the pancreatic cancer patients was very heterogeneous, with patients with all-stage tumors included in analyses. Recently, the SPARC expression was evaluated in resected PDAC patients treated with gemcitabine using the results from the CONKO-001 study ${ }^{12}$. In that study, Sinn et al. evaluated the incidence of SPARC in resectable PDAC cases using immunohistochemical methods in 109 patients and found that SPARC expression was observed in $59 \%$ of patients. In addition, Gundewar et al. measured the SPARC expression in normal pancreas, invasive adenocarcinoma, and lymph node metastasis by immunohistochemistry ${ }^{13}$. They found that high SPARC expression was observed in $77 \%$ of patients. Thus, the incidence of high SPARC expression might be relatively high in patients with resectable PDAC patients. In current study, we investigated the expression using neither Western blotting nor micro RNA. The main reason we evaluated SPARC expression by immunohistorical staining is its convenience and cost-effectiveness in practical medicine. In future, it is necessary to establish the quantitative standard for evaluation using Western blotting or micro RNA.

Regarding the relationships between SPARC expression and clinicopathological factors, Sinn et al. reported that, in an analysis of 109 patients with resected pancreatic carcinoma, there were no significant differences in the clinicopathological factors, including the UICC pT factor and lymph node status, between the patients with high and low SPARC expression ${ }^{12}$. Miyoshi et al. also reported similar results, finding no marked differences between patients with high and low SPARC expression in any clinicopathological parameters, including the UICC pT factor and lymph node metastasis ${ }^{16}$. SPARC expression therefore seems to be independent from the other clinicopathological factors.

We observed a significant difference in the OS of the patients according to the SPARC expression. Some reports have described the relationship between the SPARC expression and survival. For example, Infante et al. reported that the stromal SPARC expression is a marker of a poor prognosis in resectable pancreatic cancer ${ }^{11}$. In that report, 299 primary PDAC patients who underwent pancreaticoduodenectomy with/without adjuvant therapy were evaluated based on their resected specimens. The SPARC status in tumor and peritumoral stroma was examined by immunohistochemistry. As a result, $66.9 \%$ patients were positive for stromal SPARC, and positive SPARC expression in the stroma was associated with a poor prognosis (adjusted Cox proportional hazards: 1.89 [95\% confidence interval: 1.31-2.74]). In addition, Hann et al. reported in a meta-analysis that SPARC expression, especially in the stroma, was associated 
with a poor prognosis in patients with all-stage pancreatic cancer ${ }^{14}$.

In contrast, however, Hidalgo et al. reported that SPARC levels were not associated with treatment efficacy in patients with unresectable and metastatic pancreatic cancer in the phase III MPACT trial (OS hazard ratio: 1.019; $p=0.903)^{17}$. In that study, 256 advanced PDAC patients treated with gemcitabine with or without nab-paclitaxel chemotherapy were evaluated. The SPARC status in the stroma and plasma was examined by immunohistochemistry and enzyme-linked immune-sorbent Assay (ELISA). As a result, $28 \%$ of patients were positive for stromal SPARC, and the positive expression of SPARC in the stroma was not associated with the prognosis (hazard ratio: 1.019 [95\% confidence interval: $0.750-1.386$ ], $\mathrm{p}$ value $=0.903$ ).

In the current study, the SPARC expression was an independent prognostic factor of resected PDAC patients but not in advanced PDAC. The mechanism by which stromal SPARC expression portends a worse prognosis is unclear. The most likely hypothesis is that SPARC expression plays an important role in the proliferation of tumor stroma fibroblasts, which are related to chemo-resistance and tumor growth.

Of note, however: there was no significant difference in the RFS of the patients according to the SPARC status. Sinn et al. reported that the SPARC status is related to the recurrence ${ }^{12}$. In their report, 160 patients who participated in the CONKO-001 study were evaluated via tissue microarrays, and patients strongly expressing SPARC had a worse DFS than those weakly expressing it ( $p$ value $=0.005)$. Furthermore, there was a significant difference in the DFS according to the SPARC status in patients receiving gemcitabine adjuvant therapy. We therefore believe that the main reason for our negative result is the difference in treatment after surgery.

There are some limitations associated with this study. First, this study was a retrospective analysis that was performed at a single cancer center. Second, the post-operative treatment regimen differed among patients, with some patients receiving gemcitabine adjuvant chemotherapy and others nothing. We cannot deny the possibility that treatment variation affected the survival. Second, the present study was only analyzed the Eastern patients cohort. Therefore, similar evaluation was needed in Western cohort series.

In summary, the SPARC expression was found to be useful as a prognostic marker in pancreatic adenocarcinoma patients who underwent curative resection. The present results should be confirmed in another cohort or in a prospective study.

\section{Acknowledgments}

This work was supported by the Kanagawa Prefectural Hospitals Cancer Fund.

\section{Competing Interests}

The authors have declared that no competing interest exists.

\section{References}

1. Ferlay J, Soerjomataram I, Dikshit R, et al. Cancer incidence and mortality worldwide: sources, methods and major patterns in GLOBOCAN 2012. International journal of cancer. Mar 1 2015;136(5):E359-386

2. Rahib L, Smith BD, Aizenberg R, Rosenzweig AB, Fleshman JM, Matrisian LM. Projecting cancer incidence and deaths to 2030: the unexpected burden of thyroid, liver, and pancreas cancers in the United States. Cancer research. Jun 2014;74(11):2913-2921.

3. Oettle $H$, Post $S$, Neuhaus $P$, et al. Adjuvant chemotherapy with gemcitabine vs observation in patients undergoing curative-intent resection of pancreatic cancer: a randomized controlled trial. Jama. Jan 17 2007:297(3):267-277.

4. Neoptolemos JP, Stocken DD, Bassi C, et al. Adjuvant chemotherapy with fluorouracil plus folinic acid vs gemcitabine following pancreatic cancer resection: a randomized controlled trial. Jama. Sep 08 2010;304(10):1073-1081.

5. Uesaka K, Boku N, Fukutomi A, et al. Adjuvant chemotherapy of S-1 versus gemcitabine for resected pancreatic cancer: a phase 3, open-label, randomised, non-inferiority trial (JASPAC 01). Lancet. Jul 16 2016;388(10041):248-257.

6. Vaz J, Ansari D, Sasor A, Andersson R. SPARC: A Potential Proonostic and Therapeutic Target in Pancreatic Cancer. Pancreas. Oct 2015;44(7):1024-1035.

7. Neesse A, Michl P, Frese KK, et al. Stromal biology and therapy in pancreatic cancer. Gut. Jun 2011;60(6):861-868.

8. Neesse A, Algul H, Tuveson DA, Gress TM. Stromal biology and therapy in pancreatic cancer: a changing paradigm. Gut. Sep 2015;64(9):1476-1484.

9. Mahadevan D, Von Hoff DD. Tumor-stroma interactions in pancreatic ductal adenocarcinoma. Molecular cancer therapeutics. Apr 2007;6(4):1186-1197.

10. Prenzel KL, Warnecke-Eberz U, Xi H, et al. Significant overexpression of SPARC/osteonectin mRNA in pancreatic cancer compared to cancer of the papilla of Vater. Oncology reports. May 2006;15(5):1397-1401.

11. Infante JR, Matsubayashi $\mathrm{H}$, Sato $\mathrm{N}$, et al. Peritumoral fibroblast SPARC expression and patient outcome with resectable pancreatic adenocarcinoma. Journal of clinical oncology : official journal of the American Society of Clinical Oncology. Jan 20 2007;25(3):319-325.

12. Sinn M, Sinn BV, Striefler JK, et al. SPARC expression in resected pancreatic cancer patients treated with gemcitabine: results from the CONKO-001 study. Annals of oncology : official journal of the European Society for Medical Oncology / ESMO. May 2014;25(5):1025-1032.

13. Gundewar C, Sasor A, Hilmersson KS, Andersson R, Ansari D. The role of SPARC expression in pancreatic cancer progression and patient survival. Scandinavian journal of gastroenterology. 2015;50(9):1170-1174.

14. Han W, Cao F, Chen MB, et al. Prognostic Value of SPARC in Patients with Pancreatic Cancer: A Systematic Review and Meta-Analysis. PloS one. 2016;11(1):e0145803.

15. Scoazec JY, Sabourin JC. [2010: The seventh edition of the TNM classification]. Annales de pathologie. Feb 2010;30(1):2-6.

16. Miyoshi K, Sato N, Ohuchida K, Mizumoto K, Tanaka M. SPARC mRNA expression as a prognostic marker for pancreatic adenocarcinoma patients. Anticancer research. Mar 2010;30(3):867-871.

17. Hidalgo M, Plaza C, Musteanu M, et al. SPARC Expression Did Not Predict Efficacy of nab-Paclitaxel plus Gemcitabine or Gemcitabine Alone for Metastatic Pancreatic Cancer in an Exploratory Analysis of the Phase III MPACT Trial. Clinical cancer research : an official journal of the American Association for Cancer Research. Nov 1 2015;21(21):4811-4818.

18. Prenzel KL, Warnecke-Eberz U, Xi H, Brabender J, Baldus SE, Bollschweiler E, et al. Significant overexpression of SPARC/osteonectin mRNA in pancreatic cancer compared to cancer of the papilla of Vater. Oncology reports. 2006; 15: 1397-401.

19. Neuzillet C, Tijeras-Raballand A, Cros J, Faivre S, Hammel P, Raymond E. Stromal expression of SPARC in pancreatic adenocarcinoma. Cancer metastasis reviews. 2013; 32: 585-602.

20. Rossi MK, Gnanamony M, Gondi CS. The 'SPARC' of life: Analysis of the role of osteonectin/SPARC in pancreatic cancer (Review. International journal of oncology. 2016; 48: 1765-71.

21. Ormanns S, Haas M, Baechmann S, Altendorf-Hofmann A, Remold A, Quietzsch D, et al. Impact of SPARC expression on outcome in patients with advanced pancreatic cancer not receiving nab-paclitaxel: a pooled analysis from prospective clinical and translational trials. British journal of cancer. 2016; 115: 1520-9.

22. Yu XZ, Guo ZY, Di Y, Yang F, Ouyang Q, Fu DL, et al. The relationship between SPARC expression in primary tumor and metastatic lymph node of resected pancreatic cancer patients and patients' survival. Hepatobiliary \& pancreatic diseases international : HBPD INT. 2017; 16: 104-9. 
23. Arqueros C, Salazar J, Arranz MJ, Sebio A, Mora J, Sullivan I, et al. SPARC gene variants predict clinical outcome in locally advanced and metastatic pancreatic cancer patients. Medical oncology. 2017; 34: 136

24. Shintakuya R, Kondo N, Murakami Y, Uemura K, Nakagawa N, Okano K, et al. The high stromal SPARC expression is independently associated with poor survival of patients with resected pancreatic ductal adenocarcinoma treated with adjuvant gemcitabine in combination with S-1 or adjuvant gemcitabine alone. Pancreatology: official journal of the International Association of Pancreatology. 2018; 18: 191-7. 